\title{
Neurosífilis en pacientes portadores y no portadores de VIH: Descripción y comparación de dos cohortes históricas
}

\author{
Martín Lasso B., M. Elvira Balcells M., Ana Fernández S., Pablo Gaete G., Michel Serri V., Jorge Pérez G., \\ Carolina Chain A., Inés Cerón A., Clara Duque O. y Anamaría Ramírez B.
}

Hospital Dr. Sotero Del Río, Santiago, Chile Unidad de Infectología (MLB, AFS, PGG, MSV, ICA, ARB) Laboratorio Central, Laboratorio de Sífilis (CDO)

Hospital Clínico Universidad Católica de Chile, Santiago Unidad de Infectología (MEBM) Hospital Padre Hurtado Santiago, Chile Servicio de Medicina (JPG) Hospital Regional de Temuco Unidad de Infectología (CCA)

Recibido: 11 de marzo de 2009 Aceptado: 4 de octubre de 2009

Correspondencia a: Martín Lasso Barreto zoalde@yahoo.com

\section{Neurosyphilis in the patients with and without HIV infection. Description and comparison of two historical cohorts}

Neurosyphilis follows a more aggressive and different clinical course in HIV-infected patients compared to patients with normal immunity. Two historical series of patients with a diagnosis of neurosyphilis between 1995 and 2008 were compared: they included a group of 15 patients with y and 28 patients without HIV infection. Probability of neurosyphilis in patients with positive serum VDRL $(+)$ was increased in patients infected with HIV comparedto HIV (-) negative patients (OR: 62.37 IC:95\% (32.1-119.1) p value: $<0,001)$. Predominant clinical manifestations in neurosyphilis in the HIV (-) group were ocular abnormality, vascular encephalic and spinal cord lesions. In the HIV $(+)$ positive group, they were fever, ocular abnormalities and headache. There were no differences in cerebrospinal fluid characteristics between both groups. Neurosyphilis was diagnosed even in patients with blood VDRL of $<1: 32$ : $17,8 \%$ of HIV $(+)$ and $60 \%$ of HIV (-) patients. Penicillin sodium at dose $\geq$ than $18.000 .000 \mathrm{IU} /$ day IV during 14 days was the most common treatment. In patients with clinical neurosyphilis, $93 \%$ of HIV (-) group, and $54,2 \%$ of HIV (+) group had persistent neurological sequels. Three HIV $(+)$ patients died due to causes not related to neurosyphilis.

Key words: Neurosyphilis, HIV, CSF, lumbar puncture, VDRL uveitis.

Palabras clave: Neurosifilis, VIH, LCR, punción lumbar, VDRL uveitis.

\section{Introducción}

$\mathrm{L}$ a sífilis en el paciente con infección por VIH es más agresiva, de evolución más acelerada y de expresión clínica distinta que en inmunocompetentes $^{1,2}$. El compromiso del sistema nervioso central (SNC) es más frecuente y precoz, estimándose que la prevalencia de neurosífilis en pacientes infectados por VIH cursando sífilis en etapa latente va de $9,1 \%$ hasta $23,5 \% \%^{3,4}$. Por ende, el grado de sospecha de neurosífilis en estos pacientes debe ser alto y el diagnóstico buscado dirigidamente. Sin embargo, aún no se han validado con rigurosidad criterios clínicos o de laboratorio que indiquen la necesidad de realizar una punción lumbar en el individuo infectado por VIH con diagnóstico de sífilis ${ }^{5}$.

En Chile, se han reportado esporádicamente series de casos de neurosífilis en individuos inmunocompetentes ${ }^{6,7}$ y existe sólo una serie publicada de seis casos de neurosífilis en pacientes con infección por $\mathrm{VIH}^{8}$.

El presente trabajo busca establecer diferencias en el riesgo de aparición de neurosífilis en pacientes con sífilis portadores de VIH respecto de los no portadores del virus, así como las posibles variaciones en relación a sus manifestaciones clínicas, evolución y respuesta al tratamiento.

\section{Pacientes y Métodos}

El laboratorio de sífilis del Hospital Dr. Sótero del Río registra en forma sistemática los resultados de sus exámenes desde el año 1994. De allí se seleccionaron todos aquellos pacientes con un VDRL positivo en sangre $\geq 1$ : 2 . A su vez, se revisaron los registros de la base de datos en sistema Acces ${ }^{\circledR}$ de la Unidad de Infectología del establecimiento, con información desde el año 1997 y los registros manuscritos del Programa de Enfermedades de Transmisión Sexual, para seleccionar los pacientes portadores de VIH con un VDRL positivo en sangre $\geq 1: 2$. Se incluyó además en el análisis un paciente con infección por VIH y neurosífilis en control en Temuco, Región de la Araucanía.

Se cotejó la información separando en dos grupos a los pacientes: 
a) Pacientes seronegativos para VIH con VDRL positivo en sangre, del que, dado el gran número de pacientes en esta condición (25.960 casos) y en muchos de los cuales únicamente se disponía del informe del examen realizado, sin una historia clínica asociada (trabajadoras sexuales o controles rutinarios de instituciones o empresas), se seleccionaron sólo aquellos que disponían de una prueba treponémica positiva: microhemaglutinación para Treponema pallidum (MHA-TP), de modo que en todos los casos de este grupo se trató de sífilis confirmada.

b) Pacientes portadores de VIH con VDRL positivo en sangre. En este grupo no se exigió prueba treponémica confirmatoria y debido al carácter retrospectivo del presente trabajo, no se solicitó esta prueba en los casos seleccionados que aún estaban en control.

Por otro lado, se seleccionaron todos los pacientes con VDRL positivo en LCR de la base de datos del Laboratorio de Sífilis, verificando que tuvieran también un VDRL positivo en sangre. Estos pacientes fueron considerados como portadores de neurosífilis y se buscó dirigidamente la presencia de pruebas treponémicas positivas como el MHA-TP o el fluorescent treponemal antibody absorption (FTA-ABS).

Definiciones. Neurosifilis confirmada: VDRL positivo en sangre y en LCR más una prueba treponémica positiva en sangre. Neurosifilis probable: VDRL positivo en sangre y en LCR en ausencia de una prueba treponémica confirmatoria. Nuevo evento de neurosifilis: aquellos pacientes que habiendo resuelto las manifestaciones clínicas y disminuido en más de cuatro veces su dilución de VDRL en plasma, nuevamente presentaron una dilución de VDRL cuatro veces mayor al valor previo más bajo, reaparición de síntomas atribuibles a neurosífilis y un VDRL reactivo en LCR.

Se revisaron las fichas clínicas de los pacientes con criterios de neurosífilis confirmada y probable para obtener información respecto a edad, sexo, manifestaciones clínicas, análisis citoquímico del LCR, tratamiento anti-lúes recibido, evolución clínica y de laboratorio, y mortalidad. En el grupo neurosífilis e infección por VIH se obtuvo información respecto de carga viral y recuento de linfocitos CD4, uso de terapia anti-retroviral y presencia de infecciones oportunistas concomitantes al momento del diagnóstico de neurosífilis. Para determinar si existía diferencia entre los dos grupos en cuanto a riesgo de presencia de neurosífilis se aplicó un modelo caso-control no pareado, se obtuvo odds ratio con un IC 95\% y para definir la significancia estadística se uso la prueba de chi cuadrado. Para establecer si existía diferencias en las manifestaciones clínicas se aplicó la prueba exacta de Fisher y para determinar diferencias entre las variables continuas se aplicó la prueba $\mathrm{T}$ de Student. Se trabajó con un valor $\mathrm{p}<0,05$.

\section{Resultados}

Durante un período de 13 años y 6 meses (enero 1995 a junio 2008) se registraron 3.323 casos de sífilis confirmada en pacientes seronegativos para VIH. De éstos, 15 pacientes fueron diagnosticados como neurosífilis confirmada $(0,45 \%)$, de los cuales dos desarrollaron episodios recurrentes (en total 17 episodios de neurosífilis). La edad promedio de estos pacientes fue 50,2 años (rango: 35-73 años). La relación hombre: mujer fue de 14:1 (Tabla 1). Cuatro de estos 15 pacientes fueron diagnosticados entre enero de 1995 y junio de 1997 siendo ésta la razón para que existiera diferencia en los períodos de observación de los dos grupos.

Paralelamente, y en un período de 11 años (julio 1997 a junio 2008), existieron 1.562 pacientes con infección por VIH/SIDA en control en la Unidad de Infectología. De éstos, 127 pacientes presentaron un VDRL (+) durante su evolución, de los cuales 28 (22\%) desarrollaron neurosífilis, dos pacientes tuvieron dos episodios recurrentes (en total 30 episodios de neurosífilis). En 15 casos no se realizó prueba treponémica y en 13 se confirmó el diagnóstico con una prueba de MHA-TP positiva. La edad promedio de estos pacientes fue de 34,1 años (rango: 23-47 años), la relación hombre: mujer de 26:2, el promedio de linfocitos CD4 fue 177 céls $/ \mathrm{mm}^{3}$ (rango: 0-650 céls/ $\mathrm{mm}^{3}$ ) y el promedio de carga viral 572.503 copias ARN/mL (rango: 0-7.300.000). El 28,5\% de los pacientes con infección por VIH se encontraba recibiendo terapia anti-retroviral al momento del diagnóstico (Tabla 2). La neurosífilis se manifestó dentro del contexto de una sífilis latente en la mayoría de los casos y en sólo 7\% de las ocasiones (casos 5 y 20) se pudo verificar que se trataba de una neurosífilis temprana, esto es, dentro de los primeros 12 meses de diagnosticada la primo-infección luética.

La probabilidad de diagnosticar neurosífilis en aquellos individuos con VDRL (+) en sangre fue significativamente mayor en los pacientes infectados por VIH que en pacientes seronegativos para este virus (OR 62,37, IC95\% [32,1-119,1]; $\mathrm{p}<0,001)$. Dentro de las manifestaciones clínicas de neurosífilis, en el grupo seronegativo para VIH predominaron el compromiso ocular (33\%), accidente vascular encefálico (26,6\%), compromiso vascular medular $(13,3 \%)$ y fiebre $(13,3 \%)$ mientras que en el grupo con infección por VIH predominaron la fiebre $(42,8 \%)$, compromiso ocular $(39,3 \%)$, cefalea $(39,3 \%)$ y lesión vascular encefálica $(17,8 \%)$. No hubo pacientes infectados por VIH con daño medular. Sólo la fiebre fue significativamente más frecuente en el grupo infectado con VIH respecto del grupo seronegativo para VIH (p: 0,037)

El análisis citoquímico del LCR de los pacientes con neurosífilis no mostró diferencias significativas en pacientes con y sin infección por VIH (Tabla 3). En 15 muestras 


\begin{tabular}{|c|c|c|c|c|c|c|c|}
\hline Paciente & $\begin{array}{l}\text { Edad } \\
\text { (años) }\end{array}$ & $\begin{array}{l}\text { VDRL } \\
\text { Plasma/LCR } \\
\text { (1:/1:) }\end{array}$ & $\begin{array}{l}\text { Compromi- } \\
\text { so vascular } \\
\text { encefálico }\end{array}$ & $\begin{array}{l}\text { Lesión } \\
\text { medular }\end{array}$ & Cefalea & $\begin{array}{l}\text { Alteración } \\
\text { ocular }\end{array}$ & Observaciones \\
\hline 1 & 35 & $16 / \mathrm{RD}$ & $\mathrm{Si}$ & No & No & No & Infarto cerebral cápsula interna izquierda \\
\hline 2 & 45 & $8 / 2$ & No & Si & No & No & $\begin{array}{l}\text { Paraplejia fláccida con arreflexia, con dolor inicial } \\
\text { Completó } 10 \text { días de tratamiento }\end{array}$ \\
\hline 3 & 51 & $16 / 4$ & Si & No & No & No & $\begin{array}{l}\text { Infarto isquémico de ACM } \\
\text { Supuración de fístula perianal por } 20 \text { años }\end{array}$ \\
\hline 4 & 63 & $16 / 8$ & No & No & $\mathrm{Si}$ & $\mathrm{Si}$ & $\begin{array}{l}\text { Atrofia óptica bilateral } \\
\text { Dos episodios de neurosífilis }\end{array}$ \\
\hline 5 & 50 & $32 / 32$ & No & No & No & No & $\begin{array}{l}\text { Deterioro psico-orgánico, consumo de alcohol y cocaína. } \\
\text { Hidrocefalia }\end{array}$ \\
\hline 6 & 48 & $16 / 2$ & No & No & No & No & $\begin{array}{l}\text { Paraplejia fláccida aguda } \\
\text { RM imagen inflamatoria hiperintensa en D7 y D8 } \\
\text { compatible con mielitis transversa } \\
\text { Hiperestesia dorsal crónica y recuperación motriz parcial }\end{array}$ \\
\hline 7 & 51 & $4 / 16$ & $\mathrm{Si}$ & No & No & $\mathrm{Si}$ & $\begin{array}{l}\text { Hemiparesia braquiocrural izquierda, compromiso de tronco cerebral } \\
\text { Recuperación motriz post-tratamiento }\end{array}$ \\
\hline 8 & 38 & $4 / 8$ & $\mathrm{Si}$ & No & No & No & $\begin{array}{l}\text { Ataxia, vómito y mioclonias en hemicuerpo izquierdo, } \\
\text { AVE embólico vertebro-basilar y hemiparesia derecha posterior } \\
\text { Aneurisma basilar probablemente luético } \\
\text { Secuela parálisis pseudo-bulbar. Dos episodios de NS }\end{array}$ \\
\hline 9 & 39 & $32 / 8$ & No & No & $\mathrm{Si}$ & $\mathrm{Si}$ & Compromiso ocular con atrofia óptica \\
\hline 10 & 73 & $256 / 32$ & Si & $\mathrm{Si}$ & No & No & Infarto parietal \\
\hline 11 & 39 & $8 / 4$ & No & No & $\mathrm{Si}$ & Si & Papiledema bilateral en FO, uveítis posterior con corioretinitis \\
\hline 12 & 62 & $64 / 4$ & No & No & $\mathrm{Si}$ & Si & Neuroretinitis luética y vitreitis intensa ojo derecho \\
\hline 13 & 35 & $256 / 16$ & No & No & No & No & Asintomático \\
\hline 14 & 45 & $16 / 8$ & No & No & No & No & Asintomático \\
\hline 15 & 62 & $256 / 8$ & No & No & No & No & Lesiones hepáticas y pulmonares \\
\hline
\end{tabular}

de LCR de pacientes con infección por VIH se midió niveles de adenosin deaminasa, en cuatro de ellas $(26,6 \%)$, (casos 2, 7, 10 y 14), fue mayor de $7 \mathrm{UI} / \mathrm{mL}$ punto de corte establecido para la sospecha de meningitis tuberculosa. En el caso 14 en particular existió concomitantemente meningitis por Cryptococcus neoformans y una posible tuberculosis meníngea. El paciente recibió tratamiento para estas dos co-infecciones con respuesta satisfactoria.

En el grupo de pacientes seronegativos y en el grupo seropositivo para VIH, 17,6 y $20 \%$ respectivamente de los episodios de neurosífilis debutaron sin sintomatología neurológica, realizándose el diagnóstico sólo gracias al análisis del LCR establecido de rutina.

En 93,3 y 88,2\% respectivamente de los episodios de neurosífilis en pacientes con y sin infección por VIH, el tratamiento se realizó con penicilina $\mathrm{G}$ sódica en dosis diaria $\geq 18.000 .000$ UI por un total de 14 días; tres pacientes recibieron 20.000.000 UI/día de penicilina G sódica por 10 días (caso 25 grupo infectado por VIH, caso 2 y 8 grupo sin infección por VIH). Hubo un paciente con infección por VIH que fue tratado con ceftriaxona por alergia grave a penicilina (caso 7) y tuvo una respuesta satisfactoria.

En pacientes co-infectados con VIH, la mediana del VDRL en sangre al momento del diagnóstico fue de 1:80; sin embargo, 17,8\% de los pacientes (casos 9, 14, 15, 19 y 23) tenían un VDRL en plasma $<1: 32$ al momento del diagnóstico de neurosífilis. Tres de ellos (casos 9,15 


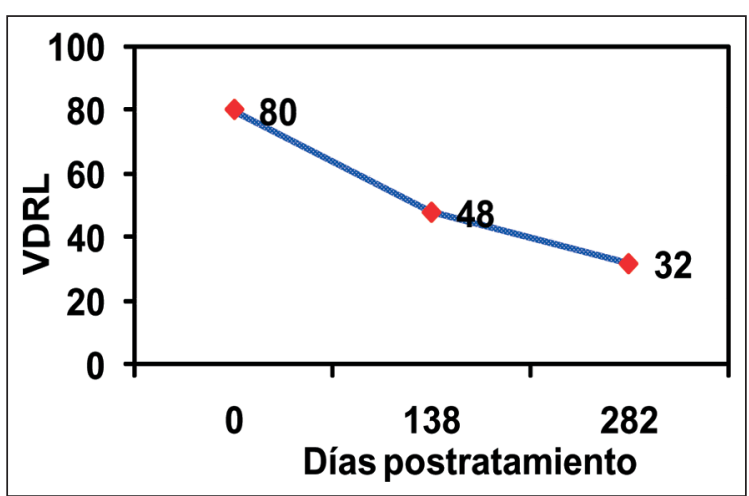

Figura 1. Descenso de la mediana de VDRL en sangre en pacientes portadores de $\mathrm{VIH}$ y neurosífilis post-tratamiento.

y 19) tuvieron manifestaciones neurológicas claras; en un paciente sus manifestaciones clínicas no pudieron ser atribuidas directamente a la neurosífilis por tener otras infecciones concomitantes del SNC (caso 14) y un paciente (caso 23) fue asintomático. Sólo en 13 pacientes se registró un segundo control de VDRL en sangre, en un tiempo promedio de 139 días después del primer examen, con una mediana de VDRL de 1:48, y en un tercer control la mediana de VDRL fue 1:32, en un tiempo promedio de 144 días respecto al segundo control (Figura 1). En pacientes no infectados por VIH la mediana del VDRL en sangre al momento del diagnóstico fue de 1:16, y 9 pacientes $(60 \%)$ tenían un VDRL en sangre $<1: 32$. El seguimiento de VDRL posterior sólo se realizó en cinco casos.

El desarrollo de secuelas neurológicas fue significativamente menor en pacientes con infección por VIH que en el grupo seronegativo (OR 0,09; 95\% IC [0,004-0,68]). De los 14 episodios de neurosífilis en individuos sin infección por VIH que presentaron compromiso neurológico clínico, se logró recuperación neurológica completa en un solo individuo (caso 7), quedando el 93\% de los pacientes con secuelas persistentes. En cambio, los 24 episodios de neurosífilis en pacientes con infección por VIH que presentaron compromiso neurológico clínico dejaron secuelas persistentes en 54,2\% de los casos.

Respecto al compromiso ocular en pacientes con infección por VIH, $38 \%$ de los 13 episodios lograron recuperación visual total, quedando $46 \%$ de los pacientes con daño visual parcial (Figura 2) y $15,5 \%$ con pérdida visual total (casos 3 y 11).

Hubo 4 (13\%) de episodios de compromiso del VIII par craneal en los pacientes portadores de VIH.

No hubo mortalidad atribuible directamente a la neurosífilis en uno ni otro grupo. Tres pacientes con infección por VIH fallecieron (casos 13, 15 y 26) posteriormente, por causas no relacionadas (Tabla 2).
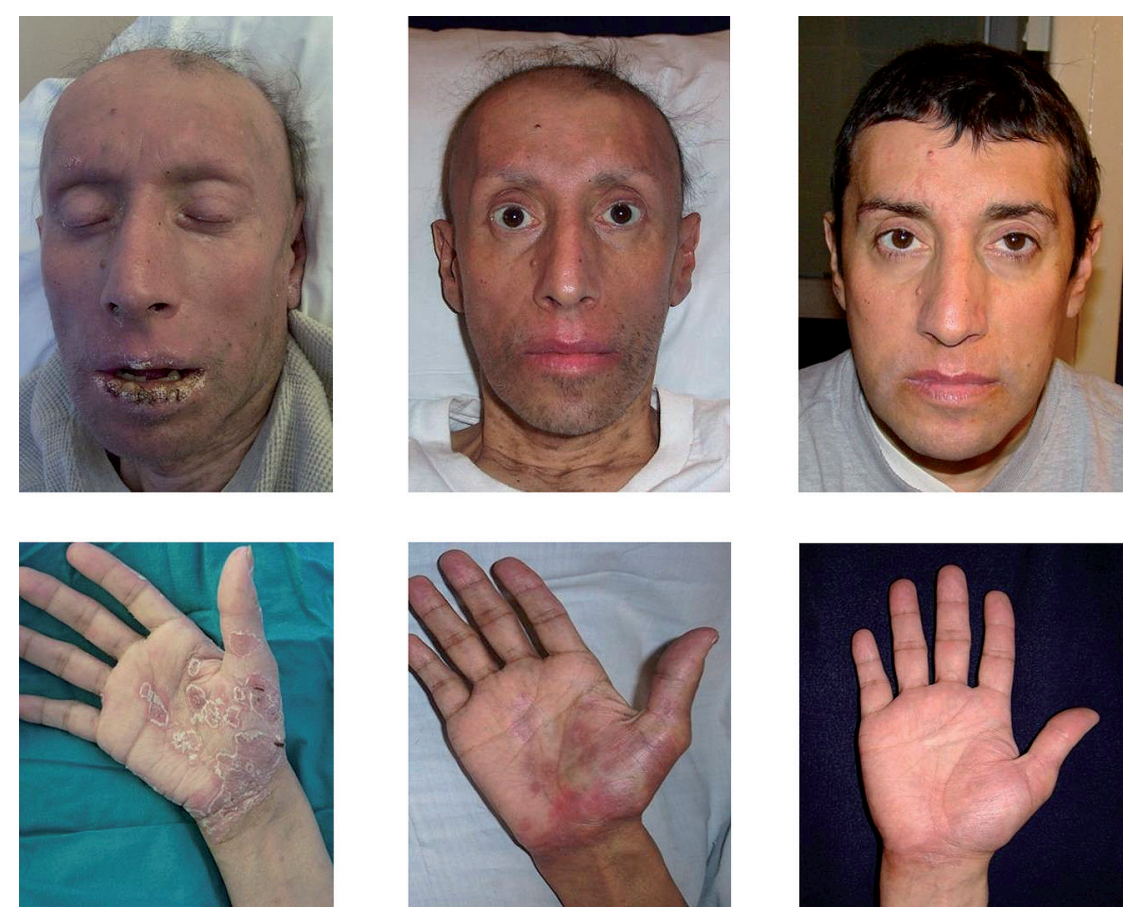

Figura 2. Paciente 1 con infección por VIH portador de neurosífilis con compromiso ocular y extensas lesiones cutáneas al momento de su diagnóstico $(A)$, después de 14 días de tratamiento con penicilina e/v (B) y luego de 3 meses de TAR (C).

\section{Discusión}

En este estudio retrospectivo se encontró una probabilidad 48 veces mayor de diagnosticar neurosífilis en los pacientes con sífilis activa y co-infección por VIH versus un grupo de pacientes inmunocompetentes. Si bien la progresión más rápida e invasión del SNC en individuos con infección por VIH es consistente con lo reportado en la literatura médica ${ }^{3,4}$, este hallazgo también puede verse influido por factores como una pesquisa activa y sistemática de neurosífilis en el paciente infectado por VIH cursando sífilis, a diferencia de los pacientes inmunocompetentes en quienes el estudio de LCR obedeció al compromiso neurológico que se presentaba en cada caso. Por otra parte, en nuestro centro, el grupo sin infección por VIH, con sífilis, está formado por un gran número de trabajadoras sexuales, en las cuales el diagnóstico de sífilis es generalmente precoz, debido a que son sometidas a controles sanitarios compulsorios que incluyen VDRL anual y tratamiento antimicrobiano inmediato de resultar positivo. En nuestros pacientes con infección por VIH no se ha incorporado aún el control rutinario de VDRL salvo cuando ingresan al programa de atención médica.

Las pruebas no treponémicas VDRL y RPR son equivalentes en el diagnóstico de sífilis activa ${ }^{2,9}$. La decisión de realizar punción lumbar en pacientes con sífilis y coinfección por VIH ha sido evaluada en otras series demostrándose que un RPR $>1: 32$ en sangre es el mejor punto 


\begin{tabular}{|c|c|c|c|c|c|c|c|c|c|c|c|}
\hline Paciente & Edad & $\begin{array}{c}\text { CD4 } \\
\text { (céls } / \mathrm{mm}^{3} \text { ) }\end{array}$ & $\begin{array}{l}\text { Carga viral VIH } \\
\text { (copias ARN/ml) }\end{array}$ & TAR & $\begin{array}{c}\text { VDRL: } \\
\text { Plasma/LCR } \\
(1: / 1:)\end{array}$ & MHA-TP & $\begin{array}{l}\text { Lesión } \\
\text { ocular }\end{array}$ & Fiebre & Cefalea & $\begin{array}{l}\text { Lesión } \\
\text { piel }\end{array}$ & $\begin{array}{l}\text { Compromiso vascu- } \\
\text { lar encefálico }\end{array}$ \\
\hline 1 & 45 & 67 & 480.000 & No & $64 / 8$ & Positivo & $\mathrm{Si}$ & $\mathrm{Si}$ & $\mathrm{Si}$ & $\mathrm{Si}$ & No \\
\hline 2 & 36 & ND & ND & No & $256 / 16$ & ND & $\mathrm{Si}$ & $\mathrm{Si}$ & $\mathrm{Si}$ & $\mathrm{Si}$ & No \\
\hline 3 & 25 & ND & ND & No & $128 / 4$ & ND & $\mathrm{Si}$ & $\mathrm{Si}$ & $\mathrm{Si}$ & No & No \\
\hline 4 & 40 & 54 & 7.300 .000 & No & $128 / 2$ & Positivo & $\mathrm{Si}$ & $\mathrm{Si}$ & No & No & No \\
\hline 5 & 30 & 185 & 0 & $\mathrm{Si}$ & $128 / 4$ & Positivo & $\mathrm{Si}$ & $\mathrm{Si}$ & No & $\mathrm{Si}$ & No \\
\hline 6 & 23 & 17 & 300.000 & No & $128 / 4$ & ND & Si & Si & No & Si & No \\
\hline 7 & 32 & 20 & 459.643 & No & $128 / 4$ & Positivo & Si & No & No & Si & No \\
\hline 8 & 27 & 81 & 210.000 & No & $128 / R D$ & ND & $\mathrm{Si}$ & No & $\mathrm{Si}$ & $\mathrm{Si}$ & No \\
\hline 9 & 29 & 247 & 73.000 & No & $16 / 2$ & Positivo & $\mathrm{Si}$ & No & No & No & No \\
\hline 10 & 26 & 335 & 570 & $\mathrm{Si}$ & $128 / R D$ & ND & $\mathrm{Si}$ & No & No & No & No \\
\hline 11 & 28 & 271 & 45.000 & No & $32 / 4$ & Positivo & $\mathrm{Si}$ & No & No & No & No \\
\hline 12 & 34 & 98 & 94.000 & No & 64 / RD & ND & No & $\mathrm{Si}$ & $\mathrm{Si}$ & No & No \\
\hline 13 & 36 & 342 & 12.000 & No & $32 / 32$ & ND & No & $\mathrm{Si}$ & $\mathrm{Si}$ & No & No \\
\hline 14 & 35 & 364 & 1.800 .000 & No & $2 / R D$ & Positivo & No & $\mathrm{Si}$ & $\mathrm{Si}$ & No & No \\
\hline 15 & 45 & ND & ND & No & $8 / 8$ & ND & No & $\mathrm{Si}$ & $\mathrm{Si}$ & No & $\mathrm{Si}$ \\
\hline 16 & 47 & 267 & 11.000 & No & 512 / RD & ND & No & $\mathrm{Si}$ & No & No & No \\
\hline 17 & 42 & ND & ND & No & $256 / 16$ & Positivo & No & No & $\mathrm{Si}$ & $\mathrm{Si}$ & $\mathrm{Si}$ \\
\hline 18 & 28 & ND & ND & $\mathrm{Si}$ & $128 / 32$ & ND & No & No & No & No & $\mathrm{Si}$ \\
\hline 19 & 34 & 22 & ND & $\mathrm{Si}$ & $16 / \mathrm{RD}$ & Positivo & No & No & $\mathrm{Si}$ & No & $\mathrm{Si}$ \\
\hline 20 & 39 & 179 & 6.200 & No & $256 / R D$ & ND & No & No & $\mathrm{Si}$ & No & No \\
\hline 21 & 37 & 650 & 0 & $\mathrm{Si}$ & $64 / 4$ & ND & No & $\mathrm{Si}$ & No & No & No \\
\hline 22 & 39 & 49 & ND & No & 64 / RD & Positivo & No & No & No & No & No \\
\hline 23 & 39 & 19 & 270.000 & No & $16 / 2$ & Positivo & No & No & No & No & No \\
\hline 24 & 28 & 292 & 0 & $\mathrm{Si}$ & $128 / 4$ & ND & No & No & No & No & No \\
\hline 25 & 33 & 300 & 94.215 & $\mathrm{Si}$ & $64 / 4$ & ND & No & No & No & No & No \\
\hline 26 & 32 & 16 & 94.000 & No & $64 / 2$ & Positivo & No & No & No & No & No \\
\hline 27 & 26 & 103 & ND & No & $512 / 2$ & ND & No & No & No & No & No \\
\hline 28 & 40 & 114 & 320.000 & No & $32 / 32$ & Positivo & No & No & & No & $\mathrm{Si}$ \\
\hline
\end{tabular}


Continuación Tabla 2

\section{Observaciones}

Uveítis con lesión nervio óptico

Recuperación visual parcial

Panuveitis. Recuperación visual total

Panuveitis y retinitis con desprendimiento de retina

Uveítis, recuperación visual parcia

Vértigo y nistagmus rotatorio

Tuberculosis concomitante

Fotofobia. Recuperación visual total

Tinitus y lesión articular. Chancro sifilítico un mes antes

Historia de meningitis por Cryptococcus sp

Dos episodios de neurosífilis

Iridociclitis con recuperación visual total en $1^{\circ}$ evento y con secuela

en $2^{\circ}$ evento post tratamiento

Ptosis y midriasis. Recuperación visual total

Corioretinits. Recuperación visual total

Epífora, catarata y movimientos extra- piramidales

Recuperación visual parcial

Dos episodios de neurosífilis con corio-retinitis. Recuperación visual parcial

Pérdida visual OI

Historia de neumonía por P. jiroveci

FMS comprometidas. Fallece

Compromiso de conciencia

Meningitis TBC y criptococóccica concomitante

CMV y bacteriemia por Providencia sp concomitante

FMS* comprometidas y hemiparesia. Fallece

Historia de hepatitis $B$

Infartos en ganglios basales cerebrales

Disartria y apraxia, infarto tronco cerebral

NS como reconstitución inmune por TAR

Accidente cerebro vascular

NS asintomática. Chancro sifilítico 9 meses antes

Hipoacusia, vértigo, nistagmus

NS asintomática. Neumonía bacteriana concomitante

NS asintomática

Historia de retinitis por CMV, neumonía por P. jiroveci y giardiasis

Asintomático

Asintomático

Neurosífilis asintomática

Historia de toxoplasmosis cerebral

Fallece

Convulsiones

Convulsiones, vértigo, infartos en protuberancia, cerebelo y hemis-

ferios. Enclaustramiento recuperado

Secuela: ataxia, disartria, hemiparesia y vejiga neurogénica

CMV: citomegalovirus
Tabla 3. Caractéristicas de citoquímico de LCR en pacientes con neurosífilis, con y sin infección por VIH

\begin{tabular}{|lcc|} 
& VIH (-) & VIH (+) \\
VDRL mediana/promedio & $8 / 10,85$ & $4 / 9,04$ \\
Recuento de células/mm $/ \mathrm{mm}^{3}$ (media) & 39,3 & 35,1 \\
Predominio & Mononuclear & Mononuclear \\
Glucosa (mg/dl) (media) & 58,0 & 45,03 \\
Proteína (g/L) (media) & 0,83 & 0,90 \\
ADA U.l/mL (media) & No disponible & 5,29 \\
\hline
\end{tabular}

de corte para decidir realizar este procedimiento, siendo muy poco probable la aparición de neurosífilis con valores por debajo de esta dilución ${ }^{5,10,11}$. No obstante, $18 \%$ de los pacientes con infección por VIH en nuestra serie tenían un VDRL en plasma $<1 / 32$ al momento del diagnóstico de neurosífilis. La guía elaborada por el Centro para el Control y Prevención de Enfermedades de Atlanta (CDC) recomienda estudiar el LCR a todo paciente infectado con VIH con sífilis latente o tardía, sífilis de duración desconocida, sífilis con síntomas y signos neurológicos y las fallas de tratamiento ${ }^{12}$. En nuestro centro se tiende a realizar punción lumbar a todo paciente con infección por VIH y con una prueba no treponémica positiva en sangre debido a que la mayoría de los pacientes con sífilis bajo nuestro control cumple alguno de los criterios mencionados para este procedimiento, pero además debido a que la punción lumbar tiene una frecuencia muy baja $(0,3 \%)$ de complicaciones ${ }^{13,14}$, a que un tercio de los pacientes con sífilis temprana tiene invasión treponémica del SNC independiente de su status para la infección por $\mathrm{VIH}^{15,16}$ y a que la penicilina benzatínica im, que sería el tratamiento habitual de una sífilis sin compromiso del SNC, no alcanza niveles terapéuticos en el LCR ${ }^{1}$. En una cohorte prospectiva de 41 pacientes, recientemente publicada, se establece como factores de riesgo asociados a la presencia de neurosífilis en pacientes infectados por VIH un nivel de linfocitos CD4 $<350$ céls/ $\mathrm{mm}^{3}$, RPR $>1$ : 128 y el sexo masculino ${ }^{17}$.

En el grupo de pacientes seronegativos para VIH, $60 \%$ (9/15) tenía un VDRL en sangre $<1 / 32$ al momento del diagnóstico de neurosífilis. Si bien el título de la prueba no treponémica en sangre parece también ser un pobre marcador de riesgo para neurosífilis en estos casos, la baja prevalencia de neurosífilis encontrada en los pacientes inmunocompetentes con VDRL $(+)(0,45 \%)$ hace cuestionable la punción lumbar rutinaria en ausencia de sintomatología neurológica.

El incremento de células y proteínas en LCR en pacientes con infección por VIH y neurosífilis, se encuentra en 38 a $69 \%$ de los casos según la serie comunicada ${ }^{17,18}$. En nuestro estudio, sólo $57 \%$ de los análisis citoquímicos de LCR se encontraron alterados, principalmente a 
expensas de un incremento de la celularidad. El aumento de ADA en 26,6\% de las muestras de LCR evaluadas en el grupo con infección por VIH sugiere que esta enzima no tiene la misma especificidad para el diagnóstico de meningitis tuberculosa que en pacientes sin infección por VIH y presenta falsos positivos en la neurosifilis, tal como está descrito para el linfoma del SNC, meningitis criptococóccica, meningo-encefalitis y poli-radiculopatía por citomegalovirus ${ }^{19,20}$. El diseño del estudio no permitió determinar la sensibilidad o especificidad del VDRL en LCR para el diagnóstico de neurolúes.

Una mención especial merecen los pacientes con neurosífilis y compromiso ocular, la mayoría de los cuales tuvieron una excelente respuesta al tratamiento y pasaron de la ceguera hasta la recuperación visual satisfactoria. La triada exantema, cefalea y compromiso ocular, descrita previamente $^{21}$, no fue habitual en nuestra serie. Por otra parte, el nivel de linfocitos CD4 bajo no parece ser un elemento categórico al momento de definir el riesgo de compromiso ocular y del SNC en el binomio sífilisinfección por VIH; en nuestra serie 39,1\% (9/25) de todos los pacientes y $33 \%(2 / 6)$ de aquellos con compromiso ocular tenían linfocitos-CD4 > 200 céls $/ \mathrm{mm}^{3}$ al momento de su diagnóstico, lo que coincide con lo reportado por otros autores ${ }^{21}$.

La neurosífilis además puede producir compromiso del $\mathrm{VIII}^{\circ}$ par craneal especialmente en el paciente VIH. Una serie tailandesa, retrospectiva, de 85 casos de oto-sífilis, determinó como las principales manifestaciones clínicas: pérdida auditiva $90,6 \%$, tinitus $72,9 \%$ y vértigo $52,9 \%$; no obstante, en esta serie apenas $5,4 \%$ tenía un LCR compatible con neurosífilis ${ }^{22}$.

Las anomalías del LCR en la neurosífilis deberían tender a mejorar a los seis meses, llegando a un LCR normal en dos años post-tratamiento ${ }^{12}$. Sin embargo, la normalización del LCR puede ser variable, siendo más rápida en pacientes inmunocompetentes $y$, dentro del grupo VIH, tarda más tiempo en corregirse cuando el recuento de CD4 es $<200$ céls $/ \mathrm{mm}^{323}$. Un estudio reciente sugirió que la normalización de los títulos de RPR séricos luego del tratamiento de la neurosífilis podría ser un predictor eficiente, tanto de la normalización del LCR como de la resolución del cuadro clínico, proponiéndose prescindir de la punción lumbar para evaluar el éxito terapéutico. El estudio encontró que más de $90 \%$ de los pacientes con un RPR normal a los 13 meses posteriores al tratamiento de neurosífilis tenían resolución clínica y normalización del $\mathrm{LCR}^{24}$. En nuestra cohorte de pacientes con infección por VIH se pudo observar un descenso en aproximadamente $50 \%$ de la dilución de VDRL en sangre a las 20 semanas en promedio; sin embargo, no se realizó un seguimiento de títulos sistemático en el tiempo.
El tratamiento de elección de la neurosífilis ha sido siempre penicilina $\mathrm{G}$ sódica endovenosa en altas dosis (18 a 24.000.000 UI/día) durante al menos 2 semanas, pero alternativas como ceftriaxona pueden ser válidas en caso de alergia o falla terapéutica ${ }^{25}$.

Por último, es importante señalar que lograr un diagnóstico y tratamiento oportuno de la neurosífilis es fundamental ya que 54 a $93 \%$ de los casos presentados tuvieron secuelas neurológicas irreversibles. Es llamativo que el porcentaje de secuelas fue significativamente mayor en el grupo de pacientes sin infección por VIH/SIDA que en el grupo con esta infección. Esto pudiera deberse a un diagnóstico más tardío ya que la punción lumbar sólo se realizó en caso de sintomatología neurológica en el grupo de no portadores de VIH

El presente trabajo refuerza la importancia de la búsqueda activa de la neurosífilis en el binomio infección por VIH-sífilis, dada la alta prevalencia de neurosífilis asintomática y con títulos bajos de VDRL en sangre en estos pacientes. Lamentablemente, pese a ser una enfermedad tratable en forma exitosa con penicilina, sigue existiendo un alto porcentaje de secuelas neurológicas, lo cual refuerza la necesidad de llegar a un diagnóstico de manera más precoz y considerar el inicio de tratamiento una urgencia.

\section{Resumen}

La neurosífilis se desarrolla de un modo más agresivo y con un perfil clínico distinto en el paciente infectado por VIH respecto de aquel que no lo está. Se comparan dos series históricas de pacientes con neurosífilis, entre 1995 y 2008: un grupo de 28 pacientes portadores de VIH y otro de 15 pacientes seronegativos para VIH. La probabilidad de encontrar neurosífilis en pacientes con VDRL positivo en sangre fue mayor en los pacientes con infección por VIH respecto de los seronegativos para VIH (OR: 62,37 IC: $95 \%(32,1-119,1) \mathrm{p}:<0,001)$. Las manifestaciones clínicas predominantes en el grupo seronegativos para VIH fueron: alteraciones oculares, vascular-encefálicas y medulares; en el grupo con infección por VIH: fiebre, compromiso ocular y cefalea. No existieron diferencias en las características del análisis citoquímico de LCR entre ambos grupos. La punción lumbar se realizó con un VDRL en sangre $<1: 32$ en $17,8 \%$ de los pacientes con infección por VIH y en $60 \%$ de los seronegativos. El tratamiento más habitual fue penicilina $\mathrm{G}$ sódica a dosis $\geq 18.000 .000$ $\mathrm{UI} /$ dia iv durante 14 días. De los pacientes con neurosífilis clínica, 93\% del grupo seronegativo para VIH y 54,2\% del grupo con infección por VIH tuvieron secuelas neurológicas persistentes. Tres pacientes infectados por VIH fallecieron por causa no atribuible a la neurosífilis. 


\section{Referencias}

1.- Musher D M, Hamill R J, Baugho R E. Effect of human immunodeficiency virus (HIV) infection on the course of syphilis and on the response to treatment. Ann Intern Med 1990; 113: 872-81.

2.- Tramont E C. Treponema pallidum (Syphilis) En Mandell, Douglas \& Bennett's Principles and Practice of Infectious Diseases Mandell G, Bennett J, Dolin R, eds. 6th ed. 2005. Elsevier. Philadelphia. pp 2768-85.

3.- Holtom P D, Larsen R A, Leal M E, Leedom J M. Prevalence of neurosyphilis in human immunodeficiency virus infected patients with latent syphilis. Am J Med 1992; 93: 9-12.

4.- Bordon J, Martínez-Vázquez C, Álvarez M, Miralles C, Ocampo A, de la Fuente-Aguado $\mathrm{J}$, et al. Neurosyphilis in HIV-infected patients. Eur J Clin Microbiol Infect Dis 1995; 14: 864-9.

5.- Marra C M, Maxwell C L, Smith S L, Lukehart S A, Rompalo A M, Eaton M, et al. Cerebrospinal fluid abnormalities in patients with syphilis. Association with clinical and laboratory features. J Infect Dis 2004; 189: 369-76.

6.- Barrientos N. Aspectos actuales de la neurolúes. Estudio de 15 pacientes. Rev Chil NeuroPsiquiatr 1983; 21: 54-60.

7.- Rivera E, Codjambassis L, Cabello F. Neurosífilis: estudio de 19 casos en Valparaíso, Chile. Rev Chil Neuro-Psiquiatr 2002; 40: 253-7.

8.- Silva C, Agar A M, Guzmán M A, Thompson L, Sepúlveda C. Neurosífilis e infección por virus de inmunodeficiencia humana (VIH-1). Experiencia con 6 casos. Rev Méd Chile 1994; 122 (12): 1393-7.

9.- Castro R, Prieto E S, da Luz Martins Pereira F. Nontreponemal test in the diagnosis of neuro syphilis: an evaluation of the Venereal Disease Research Laboratory (VDRL) and the Rapid Plasma Reagin (RPR) tests. Clin Lab Anal 2008;
22 (4): $257-61$.

10.- Libois A, De Wit S, Poll B, García F, Florence E, Del Río A, et al. HIV and syphilis: when to perform a lumbar puncture. Sexually Trans Dis 2007; 34 (3): 141-4.

11.- Ghanem K G, Moore R D, Rompalo A M, Erbelding E J, Zenilman J M, Gebo K A. Lumbar pucture in HIV infected patients with syphilis and no neurologic symptoms. Clin Infect Dis 2009; 48 (6): 816-21.

12.- Workowski K A, Berman S M. Sexually Transmitted Diseases Treatment Guidelines, 2006. MMWR Morbid Mortal Wkly Rep 2006 August 4; 55 (RR-11): 1-94.

13.- Sempere A P, Berenguer-Ruiz L, LezcanoRodas M, Mira-Berenquer F, Waez M. Punción lumbar: sus indicaciones, contraindicaciones, complicaciones y técnica. Rev Neurol 2007; 45 (7): 433-6.

14.- Wiesel J, Rose D N, Silver A L, Sacks H S, Bernstein R H. Lumbar puncture in asymptomatic late syphilis. An analysis of the benefits and risks. Arch Intern Med 1985; 145 (3): 465-8.

15.- Schofer H, Imhof M, Thoma-Greber E, Rockmeyer N H, Hartmann M, Gerken G, et al. Active syphilis in HIV infection: a multicentre retrospective survey. The German AIDS Study Group (GASG). Genitourin Med 1996; 72: $176-81$.

16.- Rolfs R T, Joesoef M R, Hendershot E F, Rompalo A M, Augenbraun M H, Chiu M, et al. A randomized trial of enhanced therapy for early syphilis in patients with and without human immunodeficiency virus infection. The Syphilis and HIV Study Group. N Engl J Med 1997; 337: 307-14.

17.- Ghanem K G, Moore R D, Rompalo A M, Erbelding E J, Zenilman J M, Gebo K A. Neurosyphilis in a clinical cohort of HIV-1 infected patients. AIDS 2008; 22 (10): 1145-51. 18.- Appleman M E, Marshall D W, Brey R L,
Houk R W, Beatty D C, Winn R E, et al Cerebrospinal fluid abnormalities in patients without AIDS who are seropositive for the human immunodeficiency virus. J Infect Dis 1988; 158 (1): 193-9.

19.- Corral I, Quereda C, Navas E, Martín-Dávila P, Pérez-Elías M J, Casado J L, et al. Adenosine deaminase activity in cerebrospinal fluid of HIV infected patients: limited value for diagnosis of tuberculous meningitis. Microbiol Infect Dis 2004; 23: 471-6.

20.- Lasso M, Pérez J, Noriega LM, Albert F, González P, Malebran A. Poliradiculopatía por citomegalovirus en dos pacientes con SIDA: tratamiento exitoso con terapia antiretroviral de alto grado de actividad (TAAGA). Rev Méd Chile 2001; 129: 1061-4.

21.- Parc C, Chahed S, Patel S; Salmon-Ceron D. Manifestations and treatment of ocular syphilis during an epidemic in France. Sexually Trans Dis 2007; 34(8): 553-6.

22.- Yimtae K, Srirompotong S, Lertsukprasert K. Otosyphilis: A review of 85 cases. Otolaryngol Head Neck Surg 2007; 136: 67-71.

23.- Marra C M, Maxwell C L, Tantalo L, Eaton M, Rompalo A M, Raines C. Normalization of cerebrospinal fluid abnormalities after neurosyphilis therapy: Does HIV status matter. Clin Infect Dis 2004; 38 (7): 1001-6. Epub 2004 Mar 16.

24.- Marra C M, Maxwell C L, Tantalo L C, Sahi S K, Lukehart S A. Normalization of serum rapid plasma reagin titer predicts normalization of cerebrospinal fluid and clinical abnormalities after treatment of neurosyphilis. Clin Infect Dis, 2008; 47 (7): 893-9.

25.- Marra C M, Boutin P, McArthur J C, Horwitz S, Simpson P A, Haslett J A, et al. A pilot study evaluating ceftriaxone and penicillin $\mathrm{G}$ as treatment agents for neurosyphilis in human immunodeficiency virus-infected individuals. Clin Infect Dis 2000; 30 (3): 540-4. 